\title{
Elementary Introduction to the Relation Between Imagination Teaching Mode and Mastering of Chinese Characters-A Summary of Middle School Teaching Experience
}

\author{
S. Palizhati \\ Chercheuse Associée au Centre Recherche Linguistique Asie Oriental, Paris, France
}

\begin{abstract}
Non-heritage Chinese learners generally think it is difficult to learn Chinese characters. Especially for French middle school students, mastering Chinese characters is the first difficulty in learning Chinese. This paper mainly relies on class experience to give exposition to the role of imagination in Chinese characters teaching.
\end{abstract}

Keywords: French students, mastering of Chinese characters, imagination

\section{Introduction}

Chinese is the second or third foreign language learned at school by French students. Language learning is influenced not only by learners' cognition factors, language atmosphere, and cultural environment as well as other factors, but also by teaching methods. Therefore, skills in the teaching of Chinese as a foreign language are important, especially to teach to master Chinese characters.

One of the difficulties in teaching Chinese as a foreign language in a French context is the learning of Chinese characters, which is the base for listening, speaking, reading, and writing. In class teaching, I found that imagination plays a very important role in the teaching of Chinese as a foreign language. It does not only simplify Chinese characters learning, but also promote the mastering of Chinese characters. So, how should a Chinese teacher in a middle school arouse students' imagination?

In class, I lead students to imagine space screens, with types of the screen to be determined according to students' hobbies, and I require students to write Chinese characters on their imagined screens, which is itself a creative labor, so it is helpful to arouse students' initiative. I hope to exchange my actual teaching experiences with my colleagues and experts, so as to understand and study methods for the teaching of Chinese as a foreign language in a French context.

\section{Chinese Characters Are an Independent System}

We know that for Chinese learners in a French context, difficulties do not only include the great difference in language and culture between Chinese and French, but also exist in the uniqueness and difficulty of Chinese characters.

Just as WANG Ning (2006) said:

S. Palizhati, Ph.D., professor of Chinese at the French Lycéesince 2012, Qualified as a lecturer by the CNU of Section 15 (Chinese Language and Civilization), Associate Research Scientist at CRLAO, Paris, France. 
The relation between Chinese characters and Chinese is completely different from that between western languages and characters. To get familiar with the research object and deeply understand and comprehend the object is the precondition to generate effective methods and excellent results. ${ }^{1}$

ZHAO Jin-ming (2008) thought that "in terms of the teaching of Chinese as a foreign language in the world, Chinese character is the biggest problem and one of the biggest challenges before the learners" (p. 98). ZHAO Jin-ming (2008) also stated that,

Based on the recognition, when we take Chinese characters as an independent system for research and teaching, we should clearly recognize that the teaching of Chinese characters is one of the biggest differences in the teaching of Chinese as the second language from the teaching of Chinese as the parent language or the teaching of other phonetic alphabet character languages. (ZHAO, 2008, p. 98)

After clearly identifying the difficulties of the non-heritage Chinese learners, it should be considered when to teach and how to teach Chinese characters. About when to teach Chinese characters, opinions differ greatly, and the issue is not the topic of my discussion today. About how to teach Chinese characters, the more popular methods at present include: component first, then the whole character; Chinese character component teaching; components and radicals teaching, etc., which are all separation teaching methods (for details, see ZHAO, 2008, p. 99). Master LV Shu-xiang said: "Some psychologists did experiments, in getting to know regardless of Chinese character or phonetic alphabet characters, learners know them from the whole shape. When knowing Chinese characters, learners do not identify the strokes of a Chinese character one by one" (LV, 1983, pp. $77-78)^{2}$

To teach French middle school students who have never seen any Chinese character, my viewpoint is that at the initial stage, the method of whole character learning, knowing, and remembering should be adopted and that too detailed analysis should be avoided. After the learners have learned and remembered a few Chinese characters and have acquired some notions of the component composition, components and radicals can be carried out to expand vocabulary. In short, teaching experiment should be based on seeking the point of penetration in Chinese character teaching.

\section{Imagined Space Screen and Chinese Characters Teaching Experience}

\section{Setting Imagined Space Screen}

In the teaching of Chinese as a second language in French middle schools, I begin with French students' living environment, interests, and hobbies to lead them to set imagined space screen, which is a job as easy as lifting a finger for every French child at the times of science and new technologies. On the other hand, French students also like creative and less difficult activities.

When learning phonetic alphabet and pronunciation, I organize every student to set his own space imagination screen according to his personal hobby. In such a case, all students imagined their system in their Iphone sets at once. I encourage students to set their own space screen or various specific or virtual items to set their own space screen. Students' imagination generally goes far beyond expectation. Students' space screens include sky, star, cloud, snow-covered land, sea, beach, hill, desert, leaf, bedroom's wall, toilet's wall, sole of a shoe, coffee mug, pencil box, their own hands, hair, and computer screen, etc.

\footnotetext{
1 ZHAO Jin-ming (2008), p. 98.

2 ZHAO Jin-ming (2008), p. 99.
} 
In Step 2, I organize students to set two files in their own imagination screen. One is a Chinese character file and the other is a vocabulary file, with the files' material forms and colors to be selected by students according to their interests, but all files to be named centrally, such as character, word, etc.

In teaching practice, I find that setting an imagined space screen can encourage students to write Chinese characters in the mode they choose and whenever they like on the one hand, and can help juvenile students to learn organization and management of the memory modes on the other hand.

During learning setting of imagined space screens, I also give the students elementary knowledge of Chinese phonetic alphabet, tone, and pronunciation; at the same time, students also learn decades of Chinese characters and several simple sentences.

\section{Saving Chinese Characters in Space Screen}

In classroom, I use the book "Character" by Professor Joël Bellassen and ZHANG Peng-peng (1989) and the book "You Say" by Arnaud Arslangul, Claude Lamoureux, and Isabelle Pilletas (2010) main textbooks.

In the primary stage of teaching, my task is to help students to learn characters, especially to learn more than 50 characters, and then I guide them to the meaning of Chinese characters (which may be words themselves, too). When teaching a Chinese character, I explain the meaning of the character, then I choose as examples several words composed of characters of the learning stage, to keep practicing, as ZHAO Jin-ming said (2008, p. 100), as far as possible: Characters and words are interwoven and should be integrated as a whole for understanding. Words include characters and characters can constitute words. In every class, the teaching activity is carried out in the following order of listening, speaking, looking, remembering, repeating, recording, and telling stories.

(1) Listening: The students listen to the words and sentences to be learned first;

(2) Speaking: The students repeat the learned words and sentences after the teacher;

(3) Looking: The students look at words and sentences shown on the blackboard;

(4) Remembering: The students have proper time for using the mode of imagination stories or figurative Chinese characters to remember what they have learned, by which way even poor creative students can mechanically memorize what they have learned. The time for remembering depends on the contents and varies from five minutes to seven or ten minutes. Here I want to explain that, for some expressions in class, I often let students use words such as "taking pictures" or "scanning", etc., to replace "remember" or "learn by heart" when memorizing Chinese characters;

(5) Repeating: Several students are asked to repeat the Chinese characters learned in last class with different modes for specific students, for example, writing on the blackboard, writing with hand in the air, pronouncing a Chinese character related to the content for review, and telling stories;

(6) Telling stories of Chinese characters: Students tell "look" of Chinese characters in their imagination and tell stories related with a Chinese character;

(7) "Taking pictures": Students take pictures of one or two selected Chinese characters relying on multi-media to magnify them and "save" them in the corresponding "photo album" in the imagined space screen.

Here I feel necessary to explain some special terms I use in class. "Taking picture" means letting students first identify Chinese characters and then remember them in the form of imagination of taking picture or 
scanning. I noticed that students, when "taking picture", are excited. While learning, every Chinese character can be taken a picture of more than once.

A Chinese character can be shown in different forms in every teaching. For example, the word “你好” (Nihao, meaning "Hello") consists of two Chinese characters. So the two characters should be presented together as much as possible.

Black and white photo: When coming across a Chinese character for the first time, I tell a story and explain its meaning in French, and then I let the students listen to and repeat it after me. With the help of multi-media, I magnify the relevant Chinese character and let the students observe it, "take black and white pictures" of it and finally save it to "black and white photo album".
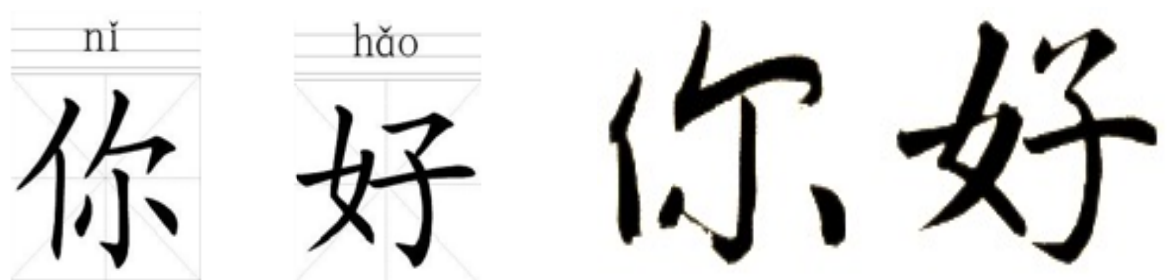

Semi-color photo: When coming across a Chinese character for the second time, I "take semi-color photos", namely using green to color the component of the Chinese characters, and ask the students to observe and remember the component, then I let them take pictures and save the picture to the "semi-color photo album".



Color photo: When coming across a Chinese character for the third time, I print the Chinese character with the green colored component on paper sheets, distribute them to the students, and let them complete the coloring process. Before that, the teacher should clearly explain the component and strokes of the Chinese character and ask the students to use only a color for a character or a component rather than color the strokes of a Chinese character with several different colors. That is one of the processes helping students to learn the strokes and component of a Chinese character. Through this process, the students can learn compositions of Chinese characters and achieve the goal of revising by a creative labor.
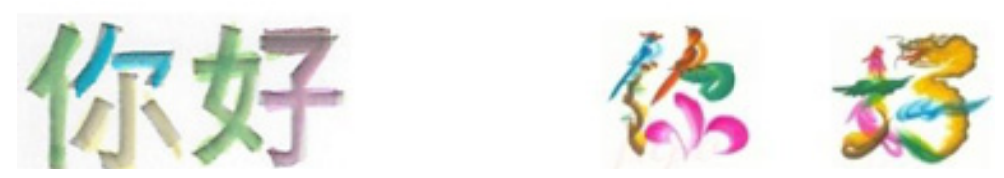

Digital photo: When coming across a Chinese character for the fourth time, I magnify it under the multi-media environment and dynamically show the character in order of the strokes, then record the strokes on the blackboard in figures, and finally let the students take a picture of the Chinese character with the order of the strokes marked, then save it in the "digital photo album". 


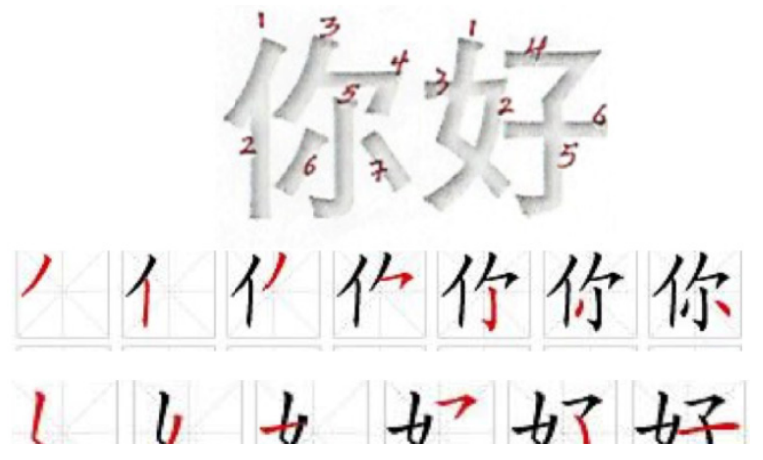

Arrow photo album: When coming across a Chinese character for the fifth time, I magnify it under the multi-media environment and dynamically show the character with the order and direction of the strokes, then I ask the students to write the Chinese character in the air after me, which is a process of training them to learn the order of the strokes. Then I use arrows to display the direction of every stroke of the Chinese character, and finally I let the students "take a picture" of the arrow-marked Chinese character and save it in the "arrow photo album".



Handwritten photo album: When coming across a Chinese character for the sixth time, I print the order of the strokes out and let the students trace model characters a few times and write them once independently. When a student correctly writes the Chinese character, I let him/her take a picture of the Chinese character he/she wrote and save it in the "handwritten" photo album.

All these are common repetitive learning processes. However, if the processes are closely combined with students' hobbies and habits, even a repeated process can make some creative results. In addition, when letting students memorize Chinese characters in the virtual form of taking a picture, I always encourage them to associate some stories related to the Chinese characters they have learned or some objects of a close shape.

After a time, I present a Chinese character they have learned and let a student tell the story from his/her imagined space screen related to the Chinese character or to the item he associated. Different students always tell different stories about the same Chinese character and a Chinese character can be presented again in the same manner after a time. It is a repetitive process, but it is not dull in students' mind.

Identifying Chinese characters does not mean to be able to write Chinese characters. To write Chinese characters gracefully needs practicing and practicing in an orderly way, step by step, and for a long time.

I think that repeating every Chinese character many times in different forms helps students to identify and learn the process of writing Chinese characters.

\section{Statistics of Teaching Practice}

I taught Chinese in three schools in the North of France. One is a private senior middle school with students of 15-22 years old, in four classes with 13-30 students per class. In this school, Chinese was taught as the third language with three courses per week. The other is a national junior middle school with students of 10-14 years old, in three classes with 16 students per class, where Chinese was taught as the second language 
with three courses per week; the third school is a national primary school, where I taught Chinese for primary school free of charge under the invitation of the municipal government, with the purpose of increasing the number of Chinese learners. These learners were at the fifth grade in primary program and at the age of 8-10 and were divided into two classes with 10-16 students per class. In the school, Chinese was taught as a part of entertainment and education with one course a week.

I noticed that more primary school and junior middle school students prefer imagined space screen than senior middle school students do and that the younger the students are, the stranger their imagined screens will be. Among the 8-13 years old students, basically every one tried to associate an item or a story corresponding to the Chinese character when learning it. For example, when learning “人” (man), “大” (big), “头” (head), and “太” (too), junior middle school students quickly associated that extra terrestrial beings' head is very big, or cartoon "Big-head Son, Small-Head Dad", etc.

The students at the age of 8-13 are basically good at "learning the whole character" and the process of character correcting and identifying is long. They cannot separate Chinese characters into components, but can only mechanically memorize characters to learn Chinese.

On the other hand, senior middle school students have a preference for separation of characters in the imagined space screen. They liked separating some Chinese characters this way or that way and recombined them. They showed shorter process of "learning by the whole character" and quickly transited to the process of separating Chinese characters into components. Moreover, they also showed the effect of the strokes of a Chinese character. Therefore, most of senior middle school students wrote Chinese characters on the space screen with their hands and most of the characters were sea, beach, sky and cloud, etc. Investigation showed that students who often wrote Chinese characters on the space screen have done any mistakes in spelling.

To sum up, all students, regardless of junior middle school students or senior middle school students, feel that the space screen is very interesting and they can write Chinese characters on the screen at any time with different colors and different tools. The space imagination screen is economical, needs no area, can accompany you all the time, and won't be forgotten at home.

\section{Conclusion}

Several years of actual tests have underlined that the imagined space screen emphasizes helping students record and sort out thinking visually. Students use the personal virtual screen to record, review, and sort out every learned character, word, or sentence. I think that the virtual screen not only conforms to the process of repetitive memorization, but also can make dull repetitive memorization process become a creative interesting flexible labor with a personal flavor. Of course, the conclusion is just based on my personal teaching experience and needs to be further tested and improved in practice. This article was discussed and researched in the Inaugural Symposium of the European Association of Chinese Teaching-Opportunities and Challenges held in Budapest, Hungary on Feb. 11-12, 2017, and supported by many colleagues, experts, and teachers of Chinese from all over the world with their valuable opinions and suggestions.

In the following teaching process, I will surely incorporate suggestions of the experts and keep deeply exploring Chinese character teaching method in a French context.

\section{References}

Arnaud, A., Claude, L., \& Isabelle, P. (2010). You say. France: DiDier. 
Joël, B. (1993). Elementary introduction to the history of the teaching of Chinese as a foreign language in France. Chinese Culture Research, 2, 139-143.

Joël, B., \& ZHANG, P. P. (1989). Character: Method and initiation to Chinese language and writing. France: The Campaign. LV, S. X. (1983). Lv Shuxiang language theory (pp. 77-78). Beijing: Commercial Press.

WANG, N. (2006). Inheritance of ancient linguistic legacy and independent innovation of linguistics. Language Science, 2.

XIN, L. Y. (2016). Word, story and culture, have you taught your children? Website administrator. Retrieved May 4, 2016 from 14:59:44; http://baby.sina.com.cn/news/list.html

ZHAO, J. M. (2008). The teaching of Chinese as the second language: Idea and mode. Chinese Teaching in the World, 1, 94-107. 